\title{
De la investigación participante a la acción política en la trasformación del Estado
}

\section{From participatory research to political action in the transformation of the State}

\author{
iD Claudia Teresa Cáceres Domínguez ${ }^{1}$
}

\section{Introducción}

\begin{abstract}
A finales del año 2008 cuando supe que tenía la oportunidad de entrar a trabajar en el Ministerio del Interior y de Justicia de Colombia, no dude en llamar para hacer llegar mi hoja de vida. Tuve la suerte (buena o mala según la mirada) de ser aceptada.
\end{abstract}

Ya había tenido la experiencia de trabajar en una institución pública del gobierno municipal, y en ese corto tiempo de trabajo, atesoré mis primeras grandes desilusiones al ver que, desde mi pequeño contrato de prestación de servicios, no podía contribuir a realizar los grandes cambios con los que habíamos soñado desde tiempo remotos.

Sin embargo, y a pesar de saber que la institucionalidad es como una araña, grande y con miles de patas, donde yo apenas había tenido una leve percepción del monstruo que era; desde el balcón de la que era mi antigua casa en Santa Marta, me lancé al vacío, respondiendo que asumiría el reto. Me dejaría tragar por aquel monstruo para verle por dentro, conocer sus múltiples ojos y poder percibir la dimensión que tiene.

El trámite para la firma del contrato fue realmente rápido. Hubo una inducción y allí conocí los primeros compañeros de la entidad, quienes en su gran mayoría entraron por carrera administrativa. Carlos, quien se encargaría de un tema de seguridad nacional, y yo éramos los únicos que veníamos a asesorar.

El primer mes de entrenamiento y conocimiento institucional fue un poco extraño, los encargos eran pocos, entonces, me di a la tarea de abrir mi primer diario de campo, en donde decidí empezar por un reconocimiento de la institución, de los compañeros de la dirección y sobretodo de sus historias de vida. Este primer ejercicio de relación fue muy importante para la gestión que me tocaría emprender, pues, en el reconocimiento de las necesidades y angustias de los demás fue dónde su amistad se volvió posible. La solidaridad se manifestó con fuerza, me sentiría una más de ellos en muy poco tiempo al compartir las profundas angustias del servicio público, y pese a que mi mirada siempre fuera la de la investigación, sobre mi espalda pesaba la firma de una resolución ministerial que me nombraba como funcionaria pública.

\footnotetext{
${ }^{1}$ Antropóloga. ORCID ID: 0000-0001-6556-4796 Correo electrónico: claudia.caceres.dominguez@usal.es
} 
Desde el piso más alto del Ministerio nos llamó, a Carlos y a mí, una asesora, quien se convertiría en mi jefe inmediata al ser nombrada Viceministra. Nos anunció nuestro nombramiento como coordinadores de grupo y los dos estábamos muy contentos y optimistas. Cuando la resolución de nombramiento estuvo lista, la Secretaria General me llamó a su despacho, leyó un texto del deber en el nombramiento del cargo y me pidió jurar, así lo hice, dos funcionarios que acompañaban el ritual me felicitaron y yo inicié con mis funciones al siguiente día.

El cargo que asumí fue la coordinación del recientemente creado grupo de consulta previa, cargo que me dio aprendizajes, dolores de cabeza, tristezas, satisfacciones, pero sobretodo, me ubicó en un punto de observación privilegiado para entender la acción del Estado y en especial del Gobierno. Vale la pena mencionar que esto ocurrió durante dos años, desde el inicio de 2009 al inicio de 2011.

Una vez, haciendo catarsis en algún documento, escribí que la resistencia debería ser activa, y allí estaba yo asumiendo las palabras que había escrito en años anteriores. Este punto de observación implicaba una participación en la toma de decisiones del Gobierno y, personalmente, fue un alto riesgo asumir esta tarea. El tiempo de dedicación era grande, al igual que las responsabilidades.

Poco a poco, conforme a los requerimientos a la entidad, según los procesos que se debían gestionar y los innumerables conflictos que había que tratar de resolver, se fueron abriendo los escenarios de discusión, las puertas de salones y despachos que revelaban las necesidades de las instituciones, los intereses económicos, los acuerdos políticos y la forma de operación de la estructura jerarquizada del poder, así como de las prácticas anómalas que genera el comportamiento clientelista $y$ burocrático.

Coordinar el tema de la consulta previa desde el Ministerio del Interior requirió un esfuerzo importante que implicó estudiar una enorme cantidad de procesos, vivenciar los conflictos en torno a la conjunción del desarrollo y la garantía de los derechos fundamentales, estudiar normas, decretos, leyes, entender el lenguaje de los abogados, de los empresarios, supervisar todos los detalles para no cometer tantos errores.

\section{Primera propuesta de metodología de acción institucional}

Para lograr tener una acción coherente era necesario contar con un equipo de trabajo, un equipo de colegas que diera las recomendaciones, orientara los procesos, cuyas ideas en el desarrollo de la labor institucional pudieran impulsarse.

Los antropólogos que participaron en esta iniciativa, teníamos claro que sería por un corto tiempo y que nuestra acción debía ser contundente y rápida. La propuesta no tenía nombre, no tenía ruta, no tenía jerarquía, pero existía, sin estas supuestas premisas de una acción coordinada. A la propuesta se unieron algunos que vieron una acción interesante, se unieron quienes veían que había posibilidades de actuación y trasformación. La acción también contaba con el conocimiento de otras disciplinas, especialmente la del derecho.

La acción consistía en generar un avance en la garantía del derecho de consulta desde dos ámbitos principalmente: el primero, desde la particularidad de cada uno de los casos, y, el segundo, generando un proceso de diálogo interinstitucional que condujo a generar una propuesta normativa y metodológica para dar un marco a la institucionalidad, realzando la participación de las comunidades y capacitando a los servidores y funcionarios en los principios del diálogo intercultural.

Paulatinamente, pero en el afán que tiene la gestión pública, nos fuimos enterando de la historia de cada uno de los procesos, pero más allá de la realización de los procedimientos que se requerían atender, debíamos hacer un esfuerzo mayor, un esfuerzo que implicara cambios en la estructura, a pesar de la inercia de las actuaciones históricas y de los prejuicios generalizados. 
Cada uno de nosotros adquirió un conocimiento particular, determinado por los escenarios de trabajo que asumió o que le fueron asignados.

Este texto busca narrar algunos aspectos de la experiencia vivida en aquellos escenarios donde se observan algunos problemas que las Ciencias Sociales, entre ellas la Antropología, han identificado en las relaciones y usos del poder en la estructura del gobierno. Entendiendo el uso del poder como sistema de presión que se materializa en una intrincada masa de normas que sustentan la acción institucional y los comportamientos sociales y culturales que se insertan en ella. Donde la propuesta de acción del grupo de trabajo estaba enmarcada, en el uso del poder como acción.

\section{La inauguración del cargo}

Cuando los líderes indígenas llegaron, se reunieron con nosotros en la sala de juntas de la Dirección de Asuntos Indígenas, reclamaban el incumplimiento del gobierno a la ley 21 de 1991, debido una inadecuada consulta previa en el Chocó. Aunque la reunión busca poner en escena las deficiencias del gobierno, nada se podía hacer; en ese punto ya todos los actores esperaban el pronunciamiento de la Corte Constitucional. Así, la Corte determinó en dicha sentencia que no se había realizado la consulta previa con las organizaciones y los representantes legítimos de las comunidades afectadas, por lo que debía rehacerse este proceso, iniciando por la identificación del área que sería afectada por el proyecto.

Cuando me citaron al salón ejecutivo para tratar el tema con altos funcionarios del gobierno, no me imaginé que la empresa tendría silla en esa reunión, pero así fue. Como yo apenas tenía unos pocos días en el cargo, opté por el silencio como estrategia para observar a los participantes y hacerme un criterio de este espacio. Sin embargo, al escuchar las generalizaciones que incriminaban a los indígenas con los grupos armados, simplemente tuve que reprocharle a un señor, ya muy mayor y cuya cabeza llena de canas no le habían dejado nada de sabiduría acerca de este asunto, enfaticé que hacer esas afirmaciones generaba vulneración a los pueblos en mención, pues los ponía en situaciones de enfrentamiento con los actores armados legales o ilegales. Esa fue la primera vez, de muchas, que el gobierno se reuniría con la empresa para buscar soluciones a las problemáticas que enfrentaba este proyecto, donde los señalamientos hacia la población indígena serían una constante.

Las discriminaciones que viven los pueblos indígenas y las comunidades afrodescendientes en estos escenarios están basadas en un concepto de superioridad de la sociedad mestiza. Esta idea de superioridad se encuentra fundada en el débil argumento de una identidad nacional homogénea, de modo que las empresas y las decisiones gubernamentales se entienden como depositarias de una identidad nacional, una identidad que supuestamente se legitima en su uniformidad, pues son ellos quienes ejecutan los proyectos y sus decisiones representan según ellos los intereses de la mayoría.

Los empresarios y altos funcionarios utilizan los argumentos de la nacionalidad y del interés general de la nación como uno de sus pilares fundamentales para la toma de decisiones. El modelo de comprensión de la nación y del Estado es más ajustado a la Constitución del 1886 que a la Constitución de 1991. Adicionalmente, el entendimiento de la "otredad", de las comunidades y pueblos, tiene un sentido de constante peligrosidad. Evidentemente, estos no son discursos que puedan estar abiertos al público en general, esas palabras se manifestaron en escenarios reducidos, privados, en escenarios de coordinación de fuerzas para el uso del poder.

Las expectativas que tienen las comunidades frente a la realización de los proyectos no son válidas para algunos, estas esperanzas se entienden como oportunistas, pues en estos espacios se cree que la identidad de los pueblos está asociada a una condición de pobreza.

La raza como categoría de representación del otro, asociada a la idea de peligrosidad, de pobreza y oportunismo, le proporcionan a estas "clases dirigentes" o sectores económicos y políticos, los argumentos que ellos mismos 
necesitan repetirse, una y otra vez, para justificar la arbitrariedad.

En los diferentes casos de trabajo, el funcionario $\mathrm{y}$ servidor público tiene una función esencial, cumplir las órdenes de las sentencias, cumplir la ley. La postura debe ser clara, el compromiso manifiesto, el cumplimiento de las normas se convierten en las palabras para el exorcismo de las "malas" intenciones. La asesoría jurídica debe ser constante, pues mucha cosas (peticiones, tutelas, hallazgos, inhabilidades, peculados, prevaricatos, agresiones, etc.) pueden ocurrir si la postura política no se respalda con el conocimiento jurídico, sensible a los temas étnicos y especializado en derecho administrativo y derecho penal.

\section{Sometimiento a la Estructura}

El clientelismo. Como en todo trabajo para acceder a laborar en el Gobierno se requiere que alguien le posibilite la entrada, bien sea porque le conoce con anterioridad y le haga partícipe de los procesos contractuales, al reconocer sus méritos profesionales, o porque se está inmerso en el comportamiento clientelista.

El clientelismo, que opera socarronamente, tiene un alto grado de tolerancia, pues permite que la acción política de la institución sea posible en ciertos escenarios. El clientelismo no es considerado internamente en el gobierno como una práctica anómala, sino que hace parte de las consecuencias propias del sistema electoral.

El sistema que, en lo local, presenta diversos comportamientos asociados a fenómenos como la compra y venta de votos, la relación con la violencia de los actores armados, entre otros asuntos, tiene una importante repercusión en la administración nacional y en la función institucional.

La "negociación" que ocurre con estos puestos de trabajo, es un intrincado sistema que no tiene únicamente que ver con un personaje, sino con el sistema de contratación pública que permite la vulneración de los derechos laborales. Este sistema exhibe incluso un retroceso importante en materia de las libertades individuales, porque las personas que llegan a trabajar al gobierno por medio de este sistema se asocian con un sistema de esclavitud o servidumbre, donde se señala que estos trabajadores tienen un "dueño". El dueño es aquel cacique político que ha solicitado los puestos de trabajo, a quien deben responder los contratistas, como engranajes dentro de las cadenas de acción política, no solo en materia electoral sino, también, en gestiones institucionales.

El problema que el clientelismo genera a la administración es enorme, pues las personas vinculadas de esta forma, en la mayor cantidad de ocasiones, no poseen un conocimiento básico en los temas que deben desarrollar. Y aunque el ejercicio de gobierno se aprende en el hacer, es necesario contar con un conocimiento experto, sobre todo si se trabaja en la garantía de los derechos fundamentales. Pero esto sería un escenario ideal, la realidad es que la institucionalidad debe optar, en el mejor de los casos, por formar en estos temas a la mayor parte de contratistas, tiempo que tarda alrededor de un año y que no asegura una adecuada formación ética.

\section{El sistema de contratación pública y los} conceptos técnicos. El sistema de contratación le permite al clientelismo ser el dueño de los derechos laborales de los trabajadores de las entidades públicas. Abusos en generación de contratos de dos y tres meses de duración, someten al profesional a unos criterios superfluos de permanencia o no en un trabajo. Este sistema potencializa en los servidores públicos la búsqueda de alianzas "económicas" con los sectores de la empresa privada, quienes aprovechan estas situaciones de vulnerabilidad laboral para igualmente comprometer la acción de estos servidores en el marco de los procesos y, en consecuencia, constriñen la acción puntual de la entidad.

Los conceptos emitidos, para la toma de decisiones institucionales, de los profesionales buscan ser modificados para responder a las necesidades de prontas gestiones o conceptos arreglados, bien sea por la empresa o por el 
ejercicio la autoridad en la estructura jerarquizada del Estado.

En estas situaciones la creatividad de los abogados en sus interpretaciones de las normas se vuelve excesivamente activa, y para poder mantener un concepto antropológico, se requiere además de un sustento jurídico que le permita tener la fuerza para establecer la línea en la gestión política.

Existe un sometimiento de los profesionales de la Antropología y de todas las otras áreas del conocimiento a un sistema que juega con sus derechos laborales y que se intensifica en una estructura jerarquizada del uso del poder. A pesar de ello los profesionales manejan situaciones políticas complejas, desestimado su rol, no solamente por ellos mismos, sino por los actores políticos y por la Academia.

Sin embargo, es comprensible esta postura ambigua, pues la acción de los profesionales se ve generalmente individualizada por la gestión de los procesos y poco o nada podemos conocer las particularidades de cada uno de ellos, las dificultades que han de resolver, tanto en el caso mismo como en sus situaciones personales. Su vulnerabilidad puede ser grande si analizamos con detenimiento esta situación y es apenas humanamente comprensible que algunos accedan a modificar sus conceptos por las presiones a las que se ven sometidos.

La escasa protección de los derechos laborales a los servidores públicos, es un costo demasiado alto para la sociedad y para cada una de las instituciones. Sumado a la falta de conocimiento experto, la acción institucional resulta plagada de errores que deben estar siendo corregidos constantemente y que, igualmente, generan unos altos costos a la institucionalidad.

\section{Conclusión}

La antropología política ha situado su actuación en la comprensión de otras culturas y su forma de gobernar. Actualmente, los antropólogos debemos también estudiar las complejidades culturales del Estado y el gobierno.
Esta corta experiencia revela la necesidad de fomentar la investigación sobre la institucionalidad, la necesidad de construir herramientas para entender estas realidades porque ellas no solo se basan en el conocimiento de las leyes, decretos y sentencias. Estas realidades necesitan ser sometidas a rigurosos procesos de investigación antropológica. Por tanto, se hace necesario construir herramientas para la investigación institucional, herramientas que implican proponer un proceso de continuidad.

Esta propuesta podrá ser acogida o no, podrá ser abandonada por su dificultad o no, lo cierto es que existen unos retos importantes para la trasformación del Estado, donde las herramientas que ha desarrollado la antropología son trascendentales para la administración pública. La Disciplina puede encontrar un espacio de investigación, trabajo y acción en estos escenarios de toma de decisiones, en la acción transformadora que inevitablemente deben tener los gobiernos para atender las necesidades de la población.

Una propuesta de este estilo implica crear una metodología de acción, que pueda generar un impacto concreto, pensado y calculado. La Investigación Acción Participante puede generar las recomendaciones que se necesitan, posicionándose apropiadamente en la escena política.

En la mayor parte de ocasiones, las personas que trabajan dentro de las instituciones públicas no evidencian las dificultades, solo viven su realidad, solo cumplen con sus funciones, por el contrario, la mirada de un investigador puede generar cambios en los discursos, recomendaciones fundamentales. La función de la Disciplina antropológica está asociada a la producción de conocimientos concretos y no podemos simplemente cerrar los ojos, ver los intereses de los mundos que están en crisis para continuar con sus propuestas.

Considero que debemos ir más allá, creo que debemos darnos la oportunidad de luchar por nuestras necesidades como gremio, en la reivindicación de nuestros derechos laborales, 
del valor de nuestro conocimiento y de las metodologías para la continua producción y acción.

Esto no se logra sino hacemos lo que mejor podemos hacer: investigar los elementos, estructuras, comportamientos, la cultura, etc., que perpetúa las desigualdades en relación a los derechos laborales, a los paradigmas homogeneizadores.

Finalmente, estoy convencida que la investigación que hacemos también puede utilizarse a favor nuestro, no debe pensarse como un acto de vanidad académica, no compromete la "objetividad científica", ni la ética profesional, sino por el contrario, esto se ha convertido en una necesidad urgente, que no solamente nos afecta como gremio, sino que afecta a la sociedad colombiana en general.

\section{Referencias}

Bricmont, J. (2005). The responsibility of the intellectual. In J. McGilvray (Ed.), The Cambridge Companion to Chomsky (pp. 280-294). Cambridge: Cambridge University Press. doi:10.1017/CCOL0521780136.015

Fals Borda, O. (1994) Investigación acción participativa. Aportes y desafíos. Bogotá, Colombia: Dimensión educativa.

Foucault, M. (1975). Vigilar y Castigar: nacimiento de la prisión. Buenos Aires, Argentina: Siglo XXI Editores.

Gellner, E. (1997). Antropología y Política. Revoluciones en el bosque sagrado. Barcelona, España: Editorial Gedisa. 